\title{
Rythmes cérébraux et codage neural de la mémoire / Brain rhythms and neural coding of memory
}

Centre interdisciplinaire de recherche en biologie (CIRB)

Michaël Zugaro

\section{OpenEdition}

\section{Journals}

Édition électronique

URL : https://journals.openedition.org/annuaire-cdf/16614

DOI : 10.4000/annuaire-cdf. 16614

ISBN : 978-2-7226-0572-5

ISSN : 2109-9227

\section{Éditeur}

Collège de France

\section{Édition imprimée}

Date de publication : 30 décembre 2020

Pagination : 674-675

ISBN : 978-2-7226-0516-9

ISSN : 0069-5580

\section{Référence électronique}

Michaël Zugaro, « Rythmes cérébraux et codage neural de la mémoire / Brain rhythms and neural coding of memory ", L'annuaire du Collège de France [En ligne], 118| 2020, mis en ligne le 01 avril 2021, consulté le 22 août 2022. URL : http://journals.openedition.org/annuaire-cdf/16614 ; DOI : https:// doi.org/10.4000/annuaire-cdf.16614 


\title{
RYTHMES CÉRÉBRAUX ET CODAGE NEURAL DE LA MÉMOIRE / BRAIN RHYTHMS AND NEURAL CODING OF MEMORY
}

\author{
Responsable : Michaël ZUGARO \\ Équipe : Nadia Benabdallah, Ariane Bochereau, Céline Boucly, Céline Drieu, \\ Ombeline HoA, Eulalie Leroux, Gabriel MAKDAH, Chantal Milleret, Virginie \\ Oberto, Marco Pompili, Susan Sara, Ralitsa Todorova, Diane Vilmer, Sidney \\ WIENER, Michaël Zugaro.
}

\section{RECHERCHE}

Page web : https://www.college-de-france.fr/site/en-cirb/zugaro.htm.

\section{Introduction}

Les «cellules de lieu» de l'hippocampe codent la position de l'animal dans l'environnement. Lorsqu'un rat se déplace, en raison de leur organisation temporelle singulière, les cellules de lieu s'activent très rapidement l'une après l'autre pour représenter la trajectoire en cours à une vitesse accélérée, ce qui favorise le renforcement de leurs connexions et pourrait sous-tendre la formation d'une première trace mnésique dans l'hippocampe. Ensuite, au cours du sommeil à ondes lentes, ces mêmes séquences se reproduisent spontanément pendant les ondulations, des oscillations hippocampiques de haute fréquence $(200 \mathrm{~Hz})$. Ces réactivations permettent un dialogue avec le cortex qui conduit à la consolidation de la mémoire, c'est-à-dire sa stabilisation à long terme - nous avons été les premiers à le démontrer lors de nos travaux précédents (Eschenko et al., 2008 ; Girardeau et al., 2009 ; Ramadan et al., 2009 ; Girardeau et al., 2014 ; Maingret et al., 2016).

Des structures sous-corticales jouent également un rôle fondamental dans ce processus, comme le striatum ou les noyaux neuromodulateurs. Nos travaux ont mis en évidence des couplages pendant le sommeil entre l'activité spontanée des neurones noradrénergiques, les ondulations hippocampiques, et les réactivations d'ensembles neuraux au niveau cortical (Mölle et al., 2006 ; Peyrache et al., 2009 ; Eschenko et al., 2012).

\section{Mise en place et réactivation des séquences hippocampiques}

Les séquences hippocampiques pourraient donc contribuer à la formation des traces mnésiques initiales, qui sont ensuite consolidées pendant le sommeil. Pour le tester, nous avons développé un protocole unique pour perturber sélectivement la formation des séquences hippocampiques au cours de l'exploration, et évaluer l'impact de cette perturbation sur les réactivations pendant le sommeil. Nous avons montré qu'en l'absence de séquences hippocampiques pendant l'exploration, on n'observe aucune réactivation pendant le sommeil. L'organisation temporelle spécifique des séquences hippocampiques est donc nécessaire à la formation des souvenirs. 


\section{Dialogue hippocampo-cortical pour la consolidation des traces mnésiques}

L'hippocampe et le cortex préfrontal médian dialoguent pendant le sommeil, ce qui permet la consolidation de la mémoire. Or, au cœur même de ce dialogue se produit une brusque interruption d'activité nommée « onde delta », et dont le rôle semble paradoxal. Nous avons découvert que, contrairement à un postulat généralement accepté, les ondes delta ne s'accompagnent pas de silence total, mais que de petits groupes de neurones corticaux poursuivent leur activité. Nos données indiquent que ces neurones, qui ont été particulièrement activés pendant l'apprentissage, participent au dialogue avec l'hippocampe, et que l'onde delta pourrait servir à les isoler des autres neurones pour éviter les interférences et faciliter la consolidation de la mémoire.

\section{Le réseau hippocampe-striatum-préfrontal dans l'apprentissage et la flexibilité cognitive}

L'apprentissage de différentes règles et la capacité à changer de stratégie en fonction du contexte mettent en jeu le cortex préfrontal, qui sous-tend certaines fonctions exécutives, ou encore le striatum, qui intervient dans la prise de décision et la motivation. Ces structures sont également impliquées dans les processus mnésiques, via leurs connexions avec l'hippocampe. Nous avons enregistré des neurones simultanément dans ces trois structures lors de l'apprentissage de deux règles différentes et du choix d'une règle en fonction du contexte. Nos résultats indiquent que les apprentissages successifs et le changement de stratégie sont soustendus par de multiples éléments du réseau cortico-striatal, et non par une seule structure. L'activité dans ces régions présente un décours temporel variable, qui dépend du niveau d'apprentissage et de la règle en cours. Enfin, la communication entre structures varie avec l'étape d'apprentissage et lors du changement de stratégie.

\section{PuBLiCATIONS}

Drieu C., Todorova R. et Zugaro M., « Nested sequences of hippocampal assemblies during behavior support subsequent sleep replay », Science, vol. 362, n 6415,2018 , p. 675679, DOI : 10.1126/science.aat2952.

TOdorova R. et Zugaro M., " Hippocampal ripples as a mode of communication with cortical and subcortical areas », Hippocampus [En ligne], 2018 ; Hippocampus, vol. 30, $\mathrm{n}^{\circ} 1$, 2020, p. 39-49, DOI : 10.1002/hipo.22997.

WIENER-VACHER S.R. et WIENER S.I., "Video head impulse tests with a remote camera system: Normative values of semicircular canal vestibulo-ocular reflex gain in infants and children », Frontiers in Neurology, vol. 8, 2017, p. 434, DOI : 10.3389/fneur.2017.00434.

MilleRET C. et BUI QUOC E., «Beyond rehabilitation of acuity, ocular alignment, and binocularity in infantile strabismus », Frontiers in Systems Neuroscience, vol. 12, 2018, DOI : 10.3389/fnsys.2018.00029. 